\title{
Comparison of thromboelastometry with procalcitonin, interleukin 6, and C-reactive protein as diagnostic tests for severe sepsis in critically ill adults
}

Michael Adamzik', Martin Eggmann ${ }^{1}$, Ulrich H Frey ${ }^{1}$, Klaus Görlinger $^{1}$, Martina Bröcker-Preuß², Günter Marggraf ${ }^{3}$, Fuat Saner ${ }^{4}$, Holger Eggebrecht ${ }^{5}$, Jürgen Peters ${ }^{1}$, Matthias Hartmann ${ }^{1 *}$

\begin{abstract}
Introduction: Established biomarkers for the diagnosis of sepsis are procalcitonin, interleukin 6, and C-reactive protein. Although sepsis evokes changes of coagulation and fibrinolysis, it is unknown whether thromboelastometry can detect these alterations. We investigated whether thromboelastometry variables are suitable as biomarkers for severe sepsis in critically ill adults.
\end{abstract}

Methods: In the observational cohort study, blood samples were obtained from patients on the day of diagnosis of severe sepsis $(n=56)$ and from postoperative patients $(n=52)$, and clotting time, clot formation time, maximum clot firmness, alpha angle, and lysis index were measured with thromboelastometry. In addition, procalcitonin, interleukin 6, and C-reactive protein levels were determined. For comparison of biomarkers, receiver operating characteristic (ROC) curves were used, and the optimal cut-offs and odds ratios were calculated.

Results: In comparison with postoperative controls, patients with sepsis showed an increase in lysis index (97\% \pm 0.3 versus $92 \pm 0.5 ; P<0.001$; mean and SEM) and procalcitonin $(2.5 \mathrm{ng} / \mathrm{ml} \pm 0.5$ versus $30.6 \pm 8.7 ; P<0.001)$. Clotformation time, alpha angle, maximum clot firmness, as well as interleukin 6 and C-reactive protein concentrations were not different between groups; clotting time was slightly prolonged. ROC analysis demonstrated an area under the curve (AUC) of 0.901 ( $\mathrm{Cl} 0.838-0.964)$ for the lysis index, and $0.756(\mathrm{Cl} 0.666-0.846)$ for procalcitonin. The calculated cut-off for the lysis index was $>96.5 \%$, resulting in a sensitivity of $84.2 \%$, and a specificity of $94.2 \%$, with an odds ratio of 85.3 (Cl 21.7 - 334.5).

Conclusions: The thromboelastometry lysis index proved to be a more reliable biomarker of severe sepsis in critically ill adults than were procalcitonin, interleukin 6, and C-reactive protein. The results also demonstrate that early involvement of the hemostatic system is a common event in severe sepsis.

\section{Introduction}

Sepsis is a common cause of death in critically ill patients, and early diagnosis is mandatory to improve the prognosis. Commonly used biomarkers like procalcitonin, C-reactive protein, and interleukin 6 are produced by the host in response to infections. However, the concentrations of these biomarkers can increase in patients with trauma or

\footnotetext{
* Correspondence: matthias.hartmann@uni-due.de

${ }^{1}$ Klinik für Anästhesiologie und Intensivmedizin, Universitätsklinikum Essen und Universität Duisburg-Essen, Hufelandstr. 55, 45130 Essen, Germany Full list of author information is available at the end of the article
}

surgery, even without infection, and, therefore, their diagnostic value in critically ill patients is far from perfect [1].

In patients with sepsis, activation of hemostasis is of marked pathophysiologic relevance, as it is associated with increased mortality [2]. As the mechanism, fibrin deposition in the vasculature, leading to ischemia and multiorgan failure, is assumed [3]. Only sparse information, however, is available on the use of thromboelastometry in sepsis. This method measures the mechanical properties of a forming clot in whole-blood samples in a time-dependent fashion and is an increasingly accepted

\section{C) Biomed Central}


point-of-care method for monitoring and therapy of hemostatic disturbances [4]. In a recent study, we demonstrated that endotoxinemia can be detected with thromboelastometry under in vitro conditions [5]. Thromboelastometric variables remained within reference ranges during the course of critically illness in 30 patients with sepsis [6]. In another study, however, early changes in thromboelastometry values were demonstrated in endotoxin-treated pigs [7].

The aim of the present study was to investigate the value of thromboelastometry variables as potential biomarkers of sepsis in critically ill adults and to compare these hemostasis-related biomarkers with the established markers procalcitonin, interleukin 6, and C-reactive protein.

\section{Materials and methods Patients}

The study was reviewed and approved by the Ethics Committee of the University Hospital Essen. In detail, informed written consent was given by both postoperative patients and probands. Informed consent of patients with sepsis was waived by the ethics committee, but written informed consent for the use of data was acquired by the surviving patients after recovery from the disease. Over a period of 2 years, 56 patients admitted to an ICU of the University Hospital of Essen were considered eligible for the study if they fulfilled the criteria for severe sepsis (sepsis group) [8]. As the second group, patients admitted to the ICU after surgery but without the criterion of sepsis were chosen (postoperative group). Groups were not matched. A detailed characterization of patients and controls is given in Table 1. As a third group, healthy probands were chosen (probands group). In all groups, whole-blood samples were subjected to thromboelastometry (ROTEM 05; Pentapharm, Germany). Samples from septic and postoperative patients were drawn within 24 hours of diagnosis and surgery, respectively. Furthermore, procalcitonin, interleukin 6 , and C-reactive protein concentrations as well as SAPS II and SOFA scores were determined in these groups at the same time $[9,10]$.

\section{Thromboelastometry}

Whole-blood coagulation properties of citrated blood samples were determined by using thromboelastometry. To exclude potential effects of heparin on coagulation, $20 \mu \mathrm{l}$ heparinase was added to the samples according to the manufacturer's recommendations (Pentapharm, München, Germany). Thereafter, samples were subjected to thromboelastometry (ROTEM 05; Pentapharm), and coagulation was initiated by addition of $\mathrm{CaCl}_{2}\left(20 \mu \mathrm{l}, 0.2 \mathrm{M} \mathrm{CaCl}_{2}\right.$, NaTEM test). Clotting time (CT), clot-formation time (CFT), maximum clot firmness (MCF), alpha angle, and the 60-minute lysis index were determined.
Table 1 Characteristics of patients with sepsis and postoperative patients

\begin{tabular}{|c|c|c|}
\hline Patient characteristics & Sepsis & Postoperative patients \\
\hline Number of patients & 56 & 52 \\
\hline Age, years & $54 \pm 17$ & $55 \pm 17$ \\
\hline Male/female & $31 / 25$ & $28 / 24$ \\
\hline Weight, kg & $79.9 \pm 23.5$ & $74.9 \pm 26.5$ \\
\hline \multicolumn{3}{|l|}{ Primary diagnosis } \\
\hline Gastrointestinal cancer & 7 & 18 \\
\hline Gastrointestinal disease & 16 & 8 \\
\hline Cancer, other & 5 & 12 \\
\hline \multirow[t]{2}{*}{ Urogenital cancer } & 3 & 9 \\
\hline & 11 & 0 \\
\hline Lung disease & 9 & 0 \\
\hline Urogenital disease & 3 & 1 \\
\hline Other diseases & 1 & 4 \\
\hline Lung cancer & 1 & 0 \\
\hline \multicolumn{3}{|l|}{ Disease severity } \\
\hline CWHD & 33 & 0 \\
\hline Mechanical ventilation, \% & 100 & 100 \\
\hline SAPS \| score & $51.4 \pm 14.9$ & $20.8 \pm 9.0$ \\
\hline SOFA score & $12.5 \pm 3.9$ & $3.85 \pm 2.6$ \\
\hline \multicolumn{3}{|l|}{ Infection type } \\
\hline Gram-positive isolates, \% & 28 & 0 \\
\hline Gram-negative isolates, \% & 49 & 0 \\
\hline Viral isolates, \% & 0 & 0 \\
\hline Fungal isolates, \% & 11 & 0 \\
\hline
\end{tabular}

Included are biometric data, primary diagnosis, disease severity, and infection type as diagnosed within 24 hours after admission. Data are given as mean and standard error of the mean. CVVHD, continuous venovenous hemodiafiltration.

\section{Assays for procalcitonin, interleukin 6, and C-reactive protein concentrations}

For the determination of procalcitonin concentration, the Liaison Brahms PCT assay (Diasorin S.p.A., Sallugia, Italy) was used. C-reactive protein was measured by using the CRP wide-range assay of the Avidia 1650 chemistry system (Bayer Healthcare LLC, Leverkusen, Germany). Interleukin 6 was determined by using an Immulite 2000 systems analyzer and reagents (Siemens Healthcare Diagnostics Products Ltd., Duisburg, Germany).

\section{Statistical analysis}

Values for the thromboelastometry variables and concentrations of procalcitonin, interleukin 6, and C-reactive protein in patients with and without severe sepsis are given as mean and standard error of the mean (SEM), as well as median and $25^{\text {th }}$ and $75^{\text {th }}$ percentiles. The Shapiro-Wilk test excluded a normal distribution for several values. Therefore, the Mann-Whitney test was used for statistical evaluation. For the comparison of biomarkers, receiver operating characteristic (ROC) curves were used, and 
these results are given as area under the curve (AUC), 95\% confidence interval (CI), and asymptotic significance ( $P$ value). Furthermore, the optimal cut-off value for each biomarker was calculated, and the corresponding sensitivities and specifities are presented. Optimal sensitivity and specificity were defined as those yielding the minimal value for $(1 \text { - sensitivity })^{2}+(1 \text { - specificity })^{2}$, as described [11]. With the calculated optimal cut-off values, the odds ratios were calculated along with the respective $95 \%$ CIs, as well as the significance values, by using the $\chi^{2}$ test. SPSS Version 16 (SPSS Inc., Chicago, IL, USA) was used for all statistical procedures, and an a priori alpha error $P$ of $<0.05$ was considered statistically significant.

\section{Results}

Thromboelastometry variables in probands and postoperative patients

In comparison with probands, postoperative patients showed an increased hemostasis potential. Thromboelastometry variables were characterized by a shorter clotting time and clot-formation time, as well as increased alpha angle and maximum clot firmness. Remarkably, the lysis index was not different in probands and postoperative patients (Table 2).

Thromboelastometry variables in critically ill patients with and without severe sepsis

In comparison with postoperative patients, sepsis patients showed an increased lysis index $(97.0 \% \pm 0.3$ versus $92.0 \pm 0.5 ; P<0.001)$ Clot-formation time, alpha angle, and maximum clot firmness were not significantly different between groups (Table 2), but the clotting time was slightly prolonged.

\section{Conventional biomarkers in critically ill patients with and} without severe sepsis

Procalcitonin, interleukin 6, and C-reactive protein concentrations were tested for differences between patients with and without sepsis. Procalcitonin concentration averaged $2.5 \mathrm{ng} / \mathrm{ml} \pm 0.5$ in postoperative patients but $30.6 \mathrm{ng} / \mathrm{ml} \pm 8.7$ in patients with severe sepsis $(P<$ $0.001)$. Neither interleukin 6 nor C-reactive protein concentrations were significantly different between patients with and without sepsis (Table 3). In both postoperative and sepsis patients, mean values for procalcitonin, interleukin 6, and C-reactive protein exceeded the reference interval by far (Table 3).

\section{Comparison of thromboelastometry variables and conventional biomarkers for the diagnosis of severe sepsis in critical ill adults}

As shown above, thromboelastometry lysis index and procalcitonin concentration were different in postoperative and sepsis patients. To further investigate the diagnostic value of these variables as potential biomarkers of severe sepsis in critical illness, a ROC curve analysis was performed. Furthermore, the 95\% confidence intervals (CI), as well as the asymptotic significance niveaus were determined. The best accuracy was yielded by the lysis index, with an AUC of 0.901 (CI 0.838 - 0.964; $P<$ 0.001 ), followed by procalcitonin concentration (AUC 0.75 ; CI $0.666-0.846 ; P<0.001$ ). The ROC curves for these variables are shown in Figure 1. Comparison of the lysis index in probands and patients with sepsis, respectively, demonstrated that the variable was capable of detecting differences between these groups with high accuracy, too (AUC 0.890; CI 0.845 - 0.977; $P<0.001$ ).

Optimal cut-off values for lysis index and procalcitonin concentration for the diagnosis of sepsis in critically ill adults

Optimal cut-off values were determined as described in the Methods section. For the lysis index, the optimum cut-off was $>96.5 \%$, resulting in a sensitivity of $84.2 \%$ and a specificity of $94.2 \%$. Applying this cut-off for the comparison of probands and sepsis patients, respectively,

Table 2 Thromboelastometry values in patients with sepsis, postoperative patients, and probands

\begin{tabular}{lllll}
\hline Thromboelastometry & $\begin{array}{l}\text { Sepsis } \\
\text { Mean [SEM] } \\
\text { Median [quartiles] }\end{array}$ & $\begin{array}{l}\text { Postoperative patients } \\
\text { Mean [SEM] } \\
\text { Median [quartiles] }\end{array}$ & $\begin{array}{l}\text { Probands } \\
\text { Mean [SEM] } \\
\text { Median [quartiles] }\end{array}$ & $\begin{array}{l}\text { Mann-Whitney test } \\
\text { Sepsis vs. postop, Sepsis vs. probands } \\
\text { Postop vs. probands }\end{array}$ \\
\hline Lysis index & $97.0 \pm 0.3$ & $92.0 \pm 0.5$ & $92.6 \pm 0.7$ & $<0.001 ;<0.001 ; 0.53$ \\
$\%$ & $98.0[97.3-98.0]$ & $92.0[90.0-95.0]$ & $93.0[91.3-95.0]$ & $0.012 ;<0.001 ;<0.001$ \\
Clotting time & $546 \pm 30$ & $434 \pm 16$ & $765 \pm 33$ & $0.085,0.003 ;<0.001$ \\
Seconds & $513[406-639]$ & $453[386-485]$ & $48.4 \pm 1.8$ & \\
Alpha-angle & $55.4 \pm 1.5$ & $59.3 \pm 1.5$ & $46.5[43.2-54.0]$ & $0.095 ; 0.01 ;<0.001$ \\
Degree & $56.0[48.0-65.0]$ & $62.0[56.0-67.0]$ & $259 \pm 17$ & $262[206-303]$ \\
Clot-formation time & $229 \pm 19$ & $193 \pm 17$ & $51.8 \pm 1.0$ & $0.858 ; 0.10 ; 0.032$ \\
Seconds & $187[136-271]$ & $166[122-196]$ & $52.0[47.5-54.8]$ & \\
Max. clot firmness & $55.4 \pm 1.5$ & $55.8 \pm 1.3$ & $57.0[50.0-62.0]$ &
\end{tabular}

Values are given as mean and standard error of the mean as well as median and $25^{\text {th }}$ and $75^{\text {th }}$ percentiles (quartiles). The Mann-Whitney test was used for statistical evaluation. 
Table 3 Conventional biomarkers of sepsis in patients with sepsis and postoperative patients

\begin{tabular}{lllll}
\hline Biomarker & $\begin{array}{l}\text { Sepsis } \\
\text { Mean [SEM] } \\
\text { Median [quartiles] }\end{array}$ & $\begin{array}{l}\text { Postoperative patients } \\
\text { Mean [SEM] } \\
\text { Median [quartiles] }\end{array}$ & Reference values & $\begin{array}{l}\text { Mann-Whitney test } \\
\text { Sepsis vs. postop } \\
P \text { value }\end{array}$ \\
\hline Procalcitonin & $30.6 \pm 8.7$ & $2.5 \pm 0.5$ & $<0.5$ (probands) & $<0.001$ \\
ng/ml & $5.5[1.5-24.3]$ & $1.4[0.4-3.3]$ & & 0.108 \\
Interleukin 6 & $1,054 \pm 426$ & $313 \pm 40$ & $0-3.4$ & 0.563 \\
pg/ml & $114[36-592]$ & $188[120-422]$ & & \\
C-reactive protein & $14.7 \pm 1.3$ & $12.5 \pm 0.7$ & $0-0.5$ & \\
mg/dl & $13.6[6.1-21.8]$ & $12.1[8.9-16.2]$ & & \\
\hline
\end{tabular}

Reference values of the biomarker assays are given. Values are given as mean and standard error of the mean as well as median and $25^{\text {th }}$ and $75^{\text {th }}$ percentiles (quartiles). The Mann-Whitney test was used for statistical evaluation.

resulted in a sensitivity of $83.9 \%$ and a specificity of $>99 \%$. The optimum cut-off for procalcitonin concentration was $>2.58 \mathrm{ng} / \mathrm{ml}$, resulting in a sensitivity of $70.2 \%$ and a specificity of $75.0 \%$.

Odds ratios for the biomarkers for the diagnosis of sepsis in critically ill patients

When applying these calculated optimum cut-off values for the biomarkers, the resulting odds ratios for the detection of severe sepsis in critically ill patients were 85.3 (CI $21.7-334.5 ; P<0.001$ ) for the lysis index and 6.3 (CI 2.7 $14.4 ; P<0.001)$ for procalcitonin concentration.

\section{Discussion}

Our results demonstrate that the thromboelastometry lysis index can discriminate intensive care patients with severe sepsis from postoperative patients and probands. Furthermore, comparison of thromboelastometry

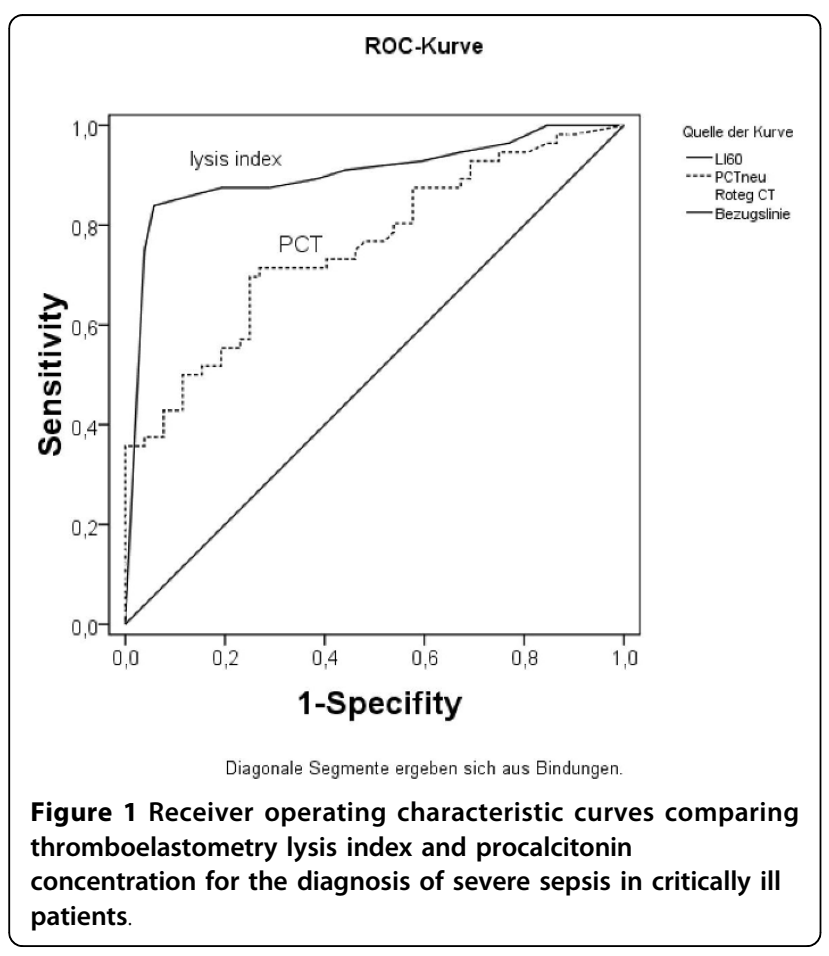

findings with the conventional biomarkers procalcitonin, interleukin 6, and C-reactive protein demonstrates a superior accuracy of the thromboelastometry lysis index in identifying patients with severe sepsis in critically ill patients. Finally, the data indicate that the fibrinolytic system is inhibited in nearly all patients with severe sepsis.

Thromboelastometry is a point-of-care method capable of determining the kinetics of clot formation and clot lysis in whole-blood samples, thereby assessing the viscoelastic properties of the clot [12]. The clotting variables obtained by thromboelastometry include the clotting time, representing the time to onset of coagulation, the clot-formation time and alpha angle, both of which describe the kinetics of clot formation, and the maximum clot firmness, describing the mechanical properties of the clot, which depends on both platelet count and fibrin polymerization. For the determination of the lysis index, the clot firmness prevailing 60 minutes after maximum clot firmness is reached, is divided by the maximum clot firmness. Thromboelastometry is widely accepted in cardiac and liver transplantation surgery $[13,14]$, but studies on its use in sepsis are sparse. Although recent data obtained in experimental sepsis and small patient cohorts suggest that sepsis-induced alterations in hemostasis might be detected with thromboelastometry, it is unknown whether thromboelastometry variables might serve as biomarkers for the diagnosis of severe sepsis $[5-7,15]$.

In Figure 2, the results obtained by both the thromboelastometry lysis index and conventional biomarkers (a) as well as the thromboelastometry clotting variables (b) are summarized. The figure demonstrates that the lysis index is not different in probands and postoperative patients but is significantly higher in patients with severe sepsis. Similarly, procalcitonin slightly increases in postoperative patients and shows a further marked increase in patients with severe sepsis. In contrast, interleukin 6 and C-reactive protein are markedly higher in postoperative patients, but no further increase is found in patients with severe sepsis. Thus, thromboelastometry lysis index and procalcitonin, but not interleukin 6 and 


\section{(a) Thrombelastometry clot lysis and conventional biomarkers}
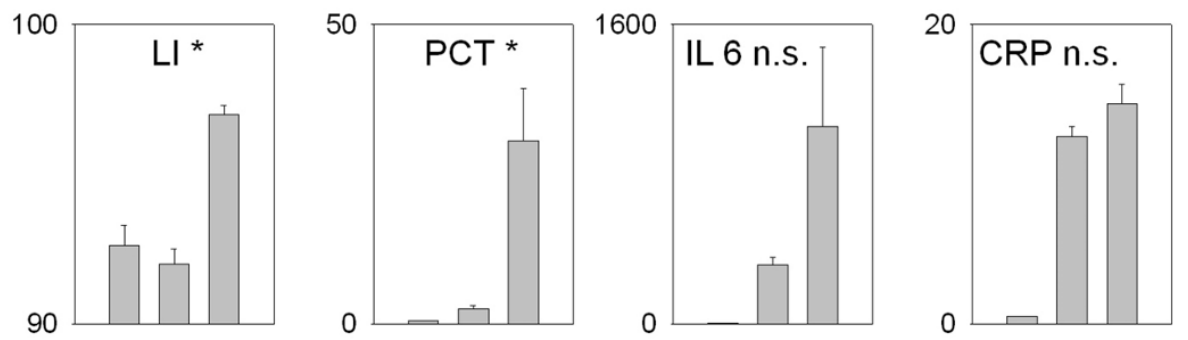

(b) Thrombelastometry clotting variables
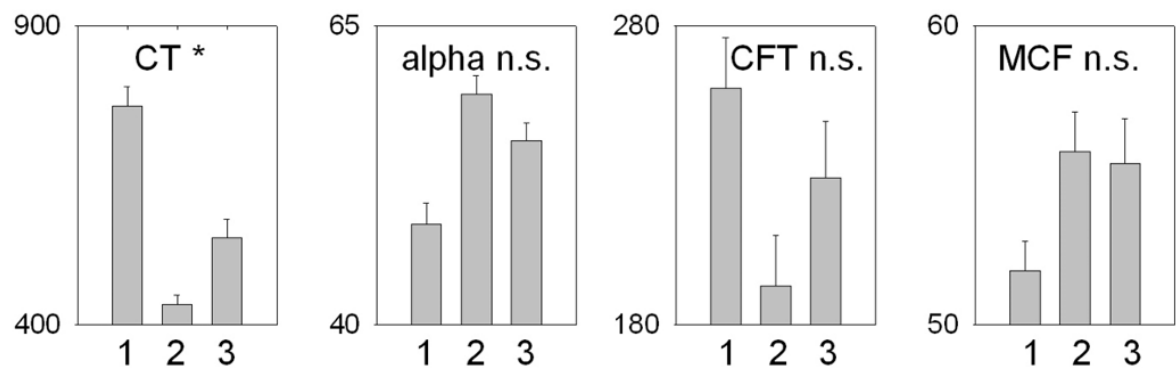

Figure 2 Thromboelastometry variables and conventional biomarkers in probands (1), postoperative patients (2), and patients with sepsis (3), respectively. Data are given as mean and standard error of the mean. The asterisks denote significant differences between postoperative and sepsis patients.

C-reactive protein, are capable of detecting patients with severe sepsis in critically ill adults. Concerning the thromboelastometry clotting variables clotting time, alpha angle, and clot-formation time, the present study demonstrates an increased hemostasis potential in postoperative patients (Figure 2b); maximum clot firmness was not different in these groups. The differences observed between postoperative patients and patients with severe sepsis were small, not significant in most cases, and thus do not allow detection of the patients with severe sepsis.

It is a main result of the present study that the thromboelastometry lysis index was increased in patients with severe sepsis in comparison with probands and postoperative patients, suggesting that the function of the fibrinolytic system is markedly inhibited. Whereas clot firmness decreased by $8 \%$ after 1 hour in patients without sepsis and in probands, clot firmness decreased by only $3 \%$ in patients with severe sepsis. The fact that the thromboelastometry lysis index was the most reliable biomarker tested for the diagnosis of severe sepsis in critically ill patients in our study demonstrates that thromboelastometry is capable of detecting changes in the fibrinolytic system in severe sepsis. Furthermore, because changes in thromboelastometry variables were seen on the day of diagnosis of severe sepsis, our data demonstrate an early involvement of the fibrinolysis system occurring in almost all patients (84.2\%) with severe sepsis. In this regard, it is important that the thromboelastometry lysis index is not different in probands and postoperative patients. Thus, an inhibition of fibrinolysis was found to be an integral part of the host response to severe infection but not to surgery.

Several reports address fibrinolysis in sepsis as well as the potential mechanisms involved [16]. Boudjeltia et al. [17] demonstrated a decrease in plasma fibrinolysis in sepsis, which was associated with organ dysfunction. As a mechanism, an increase in plasminogen activator inhibitor 1 (PAI-1), which is produced by endothelium and liver, has been demonstrated [18]. As activated protein $\mathrm{C}$ degrades PAI-1 and inhibits thrombin activable fibrinolysis inhibitor (TAFI), the decreased concentrations in activated protein $\mathrm{C}$ in sepsis may contribute to the inhibition of fibrinolysis in sepsis [19-21]. The importance of the fibrinolytic system in sepsis also has been demonstrated in genetically modified mice, showing that endotoxin-induced fibrin deposition in organs of mice deficient for tPA or uPA was more extensive than that in wild-type mice, and the opposite held true for PAI-1deficient mice [22]. Although the latter work suggests a deleterious effect of the reduced fibrinolytic rate in an endotoxin model of sepsis, others describe that local 
thrombosis/fibrin-deposition limits the survival and dissemination of microbial pathogens in mice [23]. Thus, reduced fibrinolysis in sepsis probably reduces the invasion by and the spreading of bacteria but favors disseminated intravascular coagulation, leading to organ ischemia and multiorgan failure.

The present study has limitations. The number of patients in the cohort was limited, and the sensitivity and specifity of thromboelastometry values and of conventional biomarkers for the diagnosis of sepsis might differ in other cohorts and require further studies. Furthermore, the clinical use of thromboelastometry variables as a biomarker for severe sepsis might be limited by the fact that citrated whole-blood samples have to be processed within a short time frame, and that the method is time consuming when compared with automated laboratory methods. It is a fact that the groups in the present study were heterogeneous. However, we compared several biomarker and the best biomarker, the lysis index, showed an exceedingly high odds ratio of 85.3.

\section{Conclusions}

The results of the present study demonstrate that severe sepsis is associated with reduced fibrinolysis, as evidenced by thromboelastometry. The lysis index proved to be a better biomarker for sepsis in critical illness than procalcitonin, interleukin 6 , or C-reactive protein. The fact that an inhibition of fibrinolysis occurred in nearly all patients with severe sepsis but not in postoperative patients suggests an important role of the fibrinolytic system in the pathophysiology of severe sepsis.

\section{Key messages}

- In comparison with probands and postoperative patients, the thromboelastometry lysis index is markedly increased in patients with severe sepsis.

- The thromboelastometry lysis indexed proved to be the best biomarker of sepsis in critically ill adults, followed by procalcitonin. Interleukin 6 and $C$ reactive protein were not different.

- The fact that clot lysis is reduced in almost all patients with severe sepsis suggests an important role of the fibrinolytic system in severe sepsis.

\footnotetext{
Abbreviations

AUC: area under curve; CFT: clot-formation time; Cl: confidence interval; CRP: C-reactive protein; CT: clotting time; MCF: maximum clot firmness; ROC curve: receiver operating characteristic curve.

\section{Author details}

Klinik für Anästhesiologie und Intensivmedizin, Universitätsklinikum Essen und Universität Duisburg-Essen, Hufelandstr. 55, 45130 Essen, Germany. ${ }^{2}$ Klinik für Endokrinologie, Zentrallabor Bereich Forschung und Lehre, Universitätsklinikum Essen, Hufelandstr. 55, 45122 Essen, Germany. ${ }^{3}$ Klinik für Thorax- und kardiovaskuläre Chirurgie, Universitätsklinikum und Universität
}

Duisburg-Essen, Hufelandstr. 55, 45122 Essen, Germany. ${ }^{4}$ Klinik für Allgemeinund Transplantationschirurgie, Universitätsklinikum Essen und Universität Duisburg-Essen, Hufelandstr. 55, 45122 Essen, Germany. ${ }^{5}$ Klinik für Kardiologie, Universitätsklinikum Essen und Universität Duisburg-Essen, Hufelandstr. 55, 45122 Essen, Germany.

\section{Authors' contributions}

Conception of the study was done by $M H$. MA, ME, GM, FS, HE, and MH contributed to data acquisition. $\mathrm{ME}, \mathrm{KG}$, and $\mathrm{MH}$ measured thromboelastometry variables. MB measured the conventional sepsis marker. Data were analyzed by MH, MA, UF, and JP. Drafting of the manuscript was done by $\mathrm{MH}, \mathrm{MA}$, and JP. All authors critically revised and approved the manuscript.

\section{Competing interests}

The authors declare that they have no competing interests.

Received: 27 March 2010 Revised: 27 June 2010

Accepted: 7 October 2010 Published: 7 October 2010

\section{References}

1. Uzzan B, Cohen R, Nicolas P, Cucherat M, Perret GY: Procalcitonin as a diagnostic test for sepsis in critically ill adults and after surgery or trauma: a systematic review and meta-analysis. Crit Care Med 2006, 34:1996-2003.

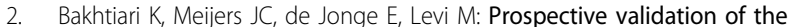
International Society of Thrombosis and Haemostasis scoring system for disseminated intravascular coagulation. Crit Care Med 2004, 32:2416-2421.

3. Gando S: Microvascular thrombosis and multiple organ dysfunction syndrome. Crit Care Med 2010, 38:S35-S42.

4. Ganter MT, Hofer CK: Coagulation monitoring: current techniques and clinical use of viscoelastic point-of-care coagulation devices. Anesth Analg 2008, 106:1366-1375.

5. Zacharowski K, Sucker C, Zacharowski P, Hartmann M: Thrombelastography for the monitoring of lipopolysaccharide induced activation of coagulation. Thromb Haemost 2006, 95:557-561.

6. Velik-Salchner C, Streif W, Innerhofer P, Maier S, Knotzer H, Pajk W, Klingler A, Mittermayr $M$, Haas T: Endotoxinemia-induced changes in coagulation as measured by rotation thrombelastometry technique and conventional laboratory tests: results of a pilot study on pigs. Blood Coagul Fibrinolysis 2009, 20:41-46.

7. Daudel F, Kessler U, Folly H, Lienert JS, Takala J, Jakob SM: : Thromboelastometry for the assessment of coagulation abnormalities in early and established adult sepsis: a prospective cohort study. Crit Care 2009, 13:R42.

8. Levy MM, Fink MP, Marshall JC, Abraham E, Angus D, Cook D, Cohen J, Opal SM, Vincent JL, Ramsay G, SCCM/ESICM/ACCP/ATS/SIS: 2001 SCCM/ ESICM/ACCP/ATS/SIS International Sepsis Definitions Conference. Crit Care Med 2003, 31:1250, 2001.

9. Le G Jr, Lemeshow S, Saulnier F: A new Simplified Acute Physiology Score (SAPS II) based on a European/North American multicenter study. JAMA 1993, 270:2957-2963.

10. Ferreira FL, Bota DP, Bross A, Mélot C, Vincent JL: Serial evaluation of the SOFA score to predict outcome in critically ill patients. JAMA 2001, 286:1754-1758.

11. Akobeng AK: Understanding diagnostic tests 3: receiver operating characteristic curves. Acta Paediatr 2007, 96:644-647.

12. Ebinger $T$, Ruland A, Lakner M, Schwaiger M: Validity, regulatory registration and approval of ROTEM thromboelastometry. Blood Coagul Fibrinolysis 2010, 21:106-107.

13. Gillies BS: Thromboelastography and liver transplantation. Semin Thromb Hemost 1995, 21(Suppl 4):45-49.

14. Dunning J, Versteegh M, Fabbri A, Pavie A, Kolh P, Lockowandt U, Nashef SA, EACTS Audit and Guidelines Committee: Guideline on antiplatelet and anticoagulation management in cardiac surgery. Eur $J$ Cardiothorac Surg 2008, 34:73-92.

15. Gonano C, Sitzwohl C, Meitner E, Weinstabl C, Kettner SC: Four-day antithrombin therapy does not seem to attenuate hypercoagulability in patients suffering from sepsis. Crit Care 2006, 10:R160.

16. Levi M, van der Poll T: Inflammation and coagulation. Crit Care Med 2010, 38:S26-S34. 
17. Boudjeltia KZ, Ollieuz S, Piagnerelli M, Biston P, Cauchie P, Vincent IL, Brohee D, Vanhaeverbeek M: Plasma fibrinolysis is related to the degree of organ dysfunction but not to the concentration of von Willebrand factor in critically ill patients. Thromb J 2009, 7:10.

18. van der Poll T, Levi M, Büller HR, van Deventer SJ, de Boer JP, Hack CE, ten Cate JW: Fibrinolytic response to tumor necrosis factor in healthy subjects. J Exp Med 1991, 174:729-732.

19. Neyrinck AP, Liu KD, Howard JP, Matthay MA: Protective mechanisms of activated protein $\mathrm{C}$ in severe inflammatory disorders. $\mathrm{Br} J$ Pharmacol 2009, 158:1034-1047.

20. Mosnier LO, Meijers JC, Bouma BN: Regulation of fibrinolysis in plasma by TAFI and protein $C$ is dependent on the concentration of thrombomodulin. Thromb Haemost 2001, 85:5-11.

21. Savioli M, Cugno M, Polli F, Taccone P, Bellani G, Spanu P, Pesenti A, lapichino G, Gattinoni L: Tight glycemic control may favor fibrinolysis in patients with sepsis. Crit Care Med 2009, 37:424-431.

22. Carmeliet P, Schoonjans L, Kieckens L, Ream B, Degen J, Bronson R, De Vos R, van den Oord JJ, Collen D, Mulligan RC: Physiological consequences of loss of plasminogen-activator gene-function in mice. Nature 1994, 368:419-424.

23. Sun $H$, Wang $X$, Degen JL, Ginsburg D: Reduced thrombin generation increases host susceptibility to group A streptococcal infection. Blood 2009, 113:1358-1364

doi:10.1186/cc9284

Cite this article as: Adamzik et al: Comparison of thromboelastometry with procalcitonin, interleukin 6 , and C-reactive protein as diagnostic tests for severe sepsis in critically ill adults. Critical Care 2010 14:R178.

\section{Submit your next manuscript to BioMed Central} and take full advantage of:

- Convenient online submission

- Thorough peer review

- No space constraints or color figure charges

- Immediate publication on acceptance

- Inclusion in PubMed, CAS, Scopus and Google Scholar

- Research which is freely available for redistribution

Submit your manuscript at www.biomedcentral.com/submit
Biomed Central 